\title{
Coenzym Q10
}

\section{Ein vitaminähnlicher Stoff für Herz, Nerven und Muskulatur}

\author{
Reinhard Saller1, Walter O. Seiler2, Christine Römer-Lüthi³, Reto Brignoli, Remy Meier ${ }^{5}$ \\ 1 UniversitätsSpital, Institut für Naturheilkunde, $\mathrm{CH}$-Zürich; ${ }^{2}$ Medizinische Universitätsklinik, Abteilung für Geriatrie, $\mathrm{CH}$-Basel; ${ }^{3}$ Ausbildungszentrum \\ Insel, CH-Bern; ${ }^{4}$ Tradyser GmbH, CH-Rüschlikon; ${ }^{5}$ Medizinische Universitätsklinik, Abteilung für Gastroenterologie, $\mathrm{CH}$-Liestal
}

D as Coenzym Q10 wurde im Jahr 1957 an der Universität Wisconsin, USA, entdeckt. Unter der Leitung von Prof. Frederick Crane wurde die Wirkungsweise der Mitochondrien in lebenden Zellen erforscht, und im Verlauf dieser Arbeiten wurden aus Rinderherzen gelbe, bis dahin unbekannte Kristalle isoliert. An der Universität von Texas gelang es der Forschergruppe um Prof. Karl FolKers, die chemische Formel der Kristalle zu entschlüsseln. Die «neue» Substanz wurde Coenzym Q10 genannt.

Coenzym $\mathrm{Q}_{10}$ gehört zu den vitaminähnlichen Stoffen, den so genannten Ubichinonen. Der Körper kann diese Stoffe selbst herstellen - im Gegensatz zu den Vitaminen. Ein Mangel an Coenzym Q10 im Organismus ist daher äusserst unwahrscheinlich und wurde bisher nicht beschrieben. Coenzym Q10 ist Bestandteil mehrerer Atmungskettenenzyme und damit an der Energiegewinnung in den Zellen beteiligt.

Ebenso wie die Vitamine $\mathrm{C}$ und $\mathrm{E}$ kann Coenzym Q10 freie Radikale neutralisieren. Dies sind Substanzen, die bei verschiedenen Stoffwechselreaktionen entstehen; besondere Bedingungen, wie etwa UV-Strahlung oder Rauchen, fördern die Bildung von freien Radikalen. Weil die freien Radikale das Gewebe schädigen, werden sie sowohl für Alterungsvorgänge als auch für Krebs, Gefässerkrankungen und weitere Leiden verantwortlich gemacht.
Hintergrund: Coenzym Q10 gehört zu den vitaminähnlichen Stoffen, den Ubichinonen. Da der Körper diese Stoffe selbst herstellen kann, ist ein Mangel an Coenzym Q10 im Organismus sehr unwahrscheinlich. Zielsetzung: Erstellung einer Risiko/Nutzen-Analyse sowie eines systematischen Review für Coenzym Q10-Supplemente. Methoden: Systematische Analyse und Bewertung von Humanstudien (prospektive Doppelblindstudien, epidemiologische und retrospektive Studien, kurzfristige biochemische/hämatologische Studien («Surrogate Markers»)) der letzten 10 Jahre aus den gängigen elektronischen Datenbanken sowie der Angaben von Standardwerken und publizierten Monographien. Ergebnisse und Schlussfolgerungen: Coenzym Q10 ist Bestandteil mehrerer Atmungskettenenzyme. Der aerobe Stoffwechsel braucht deshalb eine ausreichende Menge Coenzym Q10. Coenzym Q10 wirkt ausserdem als Antioxidans. Coenzym Q10 ist in allen Zellmembranen vorhanden. Die höchsten Konzentrationen werden in den inneren Membranen der Mitochondrien gemessen. Die Coenzym Q10-Konzentration in den einzelnen Organen ist abhängig von ihrem jeweiligen Energieumsatz. Die höchste Coenzym Q10-Konzentration findet sich in Herz und Leber. Verschiedene Nahrungsmittel, vor allem tierische Produkte, sind besonders reich an Coenzym Q10. Der Plasmaspiegel von Coenzym Q10 beträgt beim gesunden Menschen im Durchschnitt 1 umol/l. Mit dem Alter und bei bestimmten Krankheiten (Niereninsuffizienz, Asthma etc.) nehmen die Plasmaspiegel von Coenzym Q10 ab. Daraus lässt sich aber kein physiologisch bedeutsamer Mangel bzw. die Notwendigkeit einer Supplementation ableiten. Statine senken die Coenzym Q10-Spiegel. Dies kann mit Supplementen korrigiert werden, der klinische Nutzen ist aber nicht erwiesen. Coenzym Q10-Supplemente sind bei mitochondrialen enzephalomyopathischen Leiden indiziert, zum Beispiel MELAS oder hereditärem Diabetes mellitus mit Taubheit. Die Therapie mit Coenzym Q10 bei Herzinsuffizienz, Angina pectoris und Kardiomyopathien kann in Kombination mit der Standardtherapie sinnvoll sein. Vermutlich verlangsamt die Gabe von hoch dosiertem Coenzym Q10 die Progression der Parkinson-Krankheit im Anfangsstadium. Die Wirkung einer Coenzym Q10-Supplementation bei anderen Indikationen, zum Beispiel zur Leistungssteigerung in der Sportmedizin, ist noch ungenügend belegt. Bei einer Coenzym Q10-Supplementation beträgt die Tagesdosis bis zu 30 mg. Die therapeutische Dosis beträgt zwischen 60 und 1200 mg; die Indikation ist anhand der publizierten Studien vom Therapeuten empirisch festzulegen. Die Kombination von Coenzym Q10 mit anderen Antioxidantien scheint sinnvoll.

Schlüsselwörter: Coenzym Q10, Antioxidans, Herz, Nerven, Muskeln, klinische Studien

\section{Coenzyme $\mathbf{Q}_{10}$}

A vitamin-like substance for the heart, nerves and muscles

Background: Coenzyme $\mathrm{Q}_{10}$ belongs to the vitamin-like substances, the ubiquinones. As the body is able to produce these substances itself, a deficiency of coenzyme $\mathrm{Q}_{10}$ in the body is very unlikely. Objective: To draw up a risk-benefit-analysis and a systematic review for the use of Coenzyme Q10 supplements. Methods: Systematic analysis and rating of human studies (prospective double-blind studies, epidemiologic and retrospective studies, shortperiod biochemical/hematological studies ("surrogate markers")) of the last 10 years from main data-bases and of published monographs. Results and Conclusions: Coenzyme $\mathrm{Q}_{10}$ is a constituent of several respiratory chain enzymes. Aerobic metabolism therefore requires adequate quantities of coenzyme $\mathrm{Q} 10$. Coenzyme $\mathrm{Q}_{10}$ also acts as an antioxidant. Coenzyme Q10 is present in all cell membranes. The highest concentrations are measured in the inner membranes of the mitochondria. Coenzyme $\mathrm{Q} 10$ concentration in individual organs depends 
on the respective energy turnover. The highest coenzyme $\mathrm{Q}_{10}$ concentrations are found in the heart and liver. Various foodstuffs, especially animal products, are especially rich in coenzyme $\mathrm{Q}_{10}$. The plasma level of coenzyme $\mathrm{Q}_{10}$ in healthy people is on average $1 \mu \mathrm{mol} / \mathrm{l}$. The plasma levels of coenzyme $\mathrm{Q}_{10}$ fall with age and in certain diseases (renal failure, asthma, etc.). No physiologically important deficiencies or the necessity for supplementation can, however, be deduced from this. Statins reduce coenzyme Q10 level. This can be corrected with supplements, but clinical benefits have not been demonstrated. Coenzyme Q10 supplements are indicated in mitochondrial encephalomyopathic disorders, e.g. MELAS (Myopathy, Encephalopathy, Lactic Acidosis, Stroke-like Episodes) or hereditary diabetes mellitus with deafness. Treatment with coenzyme $\mathrm{Q}_{10}$ in heart failure, angina pectoris and cardiomyopathies may be worthwhile in combination with standard therapy. Presumably, the administration of high doses of coenzyme $\mathrm{Q} 10$ slows progression of Parkinson's disease in the early stages. The action of coenzyme Q10 supplementation in other indications, e.g. to improve performance in sports, has not been sufficiently confirmed. For coenzyme $\mathrm{Q}_{10}$ supplementation, the daily dose is up to $30 \mathrm{mg}$. The therapeutic dose is 60-1200 mg. The indication must be empirically determined by the therapist on the basis of published studies. Combination of coenzyme Q10 with other antioxidants appears to be rational.

Key words: Coenzyme Q10, antioxidant, heart, nerves, muscles, clinical trials

\section{Die Struktur und Synthese von Coenzym $\mathrm{Q}_{10}$}

Coenzym Q10 kann in allen Membranen nachgewiesen werden. Die Länge der Kette aus Isopren-Einheiten scheint zwischen sechs und zehn Einheiten zu variieren (Abbildung 1). Coenzym $Q_{10}$ wirkt als lipophiles Antioxidans. In dieser Eigenschaft ist es eher als Hydroxyl- denn als Superoxid-Fänger beschrieben worden. Die UbisemichinonUbichinon-Reaktion ist als prooxidative Reaktion aufzufassen. Sie dient unter anderem zur Regeneration von Tocopheryl-Radikalen innerhalb der Zellmembranen. Zur Synthese von Coenzym Q10 siehe Abbildung 2.

\section{Coenzym $\mathbf{Q}_{10}$ in den Mitochondrien}

Innerhalb der Zelle werden die höchsten Coenzym Q10-Konzentrationen in den inneren Membranen der Mitochondrien und in den Membranen des Golgiapparats gemessen (Tabelle 1). Obwohl die exogene Zufuhr von Coenzym Q10 die Blutspiegel erhöht, ist unklar, ob exogenes Coenzym $Q_{10}$ auch in den Organellen der Zellen angereichert wird. Verschiedene Nahrungsmittel, vor allem tierische Produkte, sind besonders reich an Coenzym Q10 (Tabelle 2).

Als lipophile Substanz, die aufgrund ihrer Chinonringstruktur in der Lage ist, Elektronen aufzunehmen bzw. wieder abzugeben, ist das Coenzym Q10 integraler Bestandteil der in den Mitochondrienmembranen ablaufenden Reaktionen der Atmungskette. Bei diesem Prozess werden Elektronen aus Wasserstoff unter Bildung von Wasser auf Sauerstoff übertragen. Die dabei frei werdende Energie wird teilweise durch die oxidative Phosphorylierung in Form von ATP konserviert. Das ATP steht dann als Energielieferant für die zellulären Funktionen zur Verfügung, zum Beispiel für Muskelkontraktionen, die Aufrechterhaltung von Ionengradienten etc. Coenzym $Q_{10}$ kann in der Atmungskette durch keine andere Substanz ersetzt werden. Der aerobe Stoffwechsel ist deshalb unmittelbar auf eine ausreichende Menge Coenzym Q10 angewiesen.

In der Atmungskette gibt es fünf Membran-gebundene Enzymkomplexe. Coenzym Q10 befindet sich in Komplex I, dem grössten und noch am wenigsten verstandenen Komplex, zu dem auch noch keine detaillierten Strukturdaten vorliegen. Komplex I besteht aus mindestens 43 verschiedenen Polypeptiden, von denen 7 durch die mitochondriale DNS kodiert werden.

Coenzym Q10 nimmt in der inneren Mitochondrienmembran über Flavoproteine Wasserstoff und damit zwei Elektronen auf. Diese werden einzeln an die Coenzyme von Komplex III weitergeleitet. Coenzym $\mathrm{Q}_{10}$ hat damit eine zentrale Funktion bei der Einschleusung von Wasserstoff in die Atmungskette und bei der mitochondrialen Energiegewinnung.
Coenzym Q10-Konzentration im menschlichen Körper

Coenzym Q10 kommt in allen Zellen des menschlichen Körpers vor. Die Coenzym-Q 10 -Konzentration in den einzelnen Organen ist jedoch abhängig von deren jeweiligem Energieumsatz (=Mitochondriendichte). So ist Coenzym Q10 in einigen Organen in viel höherer Konzentration vorhanden als in anderen. Die höchste Coenzym-Q10Konzentration findet sich in Herz und Leber, also den Organen, die einen sehr hohen Energiebedarf haben und die meisten Mitochondrien aufweisen. Auch in Niere und Pankreas sind die Coenzym-Q10-Konzentrationen sehr hoch.

Der Plasmaspiegel von Coenzym Q10 beträgt beim gesunden Menschen im Durchschnitt $1 \mu \mathrm{mol} / \mathrm{l}$. Die Plasmaspiegel korrelieren mit den Aktivitäten der Komplexe I und II/III in den Mitochondrien [1]. Die Faktoren, welche die Coenzym-Q10-Spiegel im Blut beeinflussen, sind in Tabelle 3 zusammengefasst.

Mit dem Alter nehmen die CoenzymQ10-Konzentrationen ab. In Herzmuskel, Pankreas, Nebennieren, Nieren und Haut (Epidermis) beträgt die Abnahme über 50 Prozent [2]. Ein $\mathrm{Zu-}$ sammenhang zwischen Koronarsklerose und verminderten Coenzym-Q10Konzentrationen konnte allerdings nicht nachgewiesen werden [3]. Bei Frauen, die über 60 Jahre alt waren, wurden Coenzym-Q10-Spiegel von 0.99 $\mu \mathrm{mol} / \mathrm{l}$ beschrieben (5.-95. Perzentile: 0.54-1.68), die auf Cholesterin bezogene Coenzym Q10-Konzentration betrug aber nur 0.16 (5.-95. Perzentile: 0.090.26) $\mu \mathrm{mol} / \mathrm{mol}$ Cholesterin [4]. Diese Werte waren unabhängig vom Alter, von der gleichzeitigen Behandlung mit Statinen und vom Körpergewicht.

Ein «Mangel» an Coenzym Q10 bzw. tiefe Plasmaspiegel wurden bei verschiedenen Pathologien beschrieben, z.B. bei der juvenilen neuronalen Zeroid-Lipofuszinose (JNCL), renaler Insuffizienz, Hämodialyse, Asthma, Asthenozoospermie etc.

In einer Studie erhielten Probanden während bis zu drei Monaten täglich Coenzym-Q10-Dosen zwischen 30 und $200 \mathrm{mg}$. Als Resultat wurden dosisabhängige Anstiege der Coenzym-Q10- 


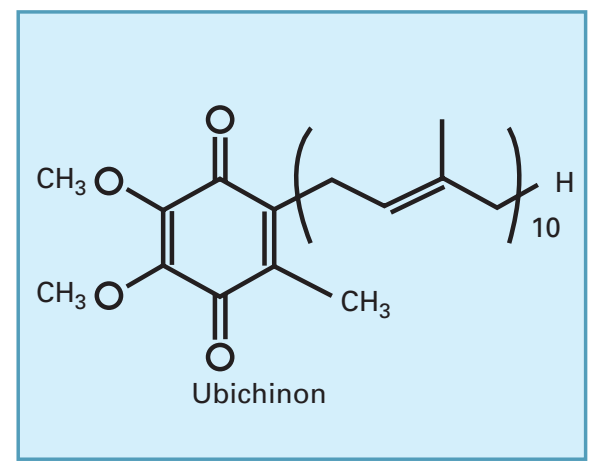

Abb. 1. Formel von Coenzym $\mathrm{Q}_{10}$ (Ubichinon).

Plasmakonzentrationen gemessen. Die Resorption von exogenem Coenzym Q10 wird durch die gleichzeitige Gabe von Vitamin E abgeschwächt [5] und die gemessenen Coenzym-Q10-Plasmakonzentrationen sind auch von der galenischen Form des Coenzym-Q10Präparats abhängig [6].

\section{Interaktionen von Coenzym Q10}

Statine hemmen die HMG-CoA-Reduktase (vergleiche Abbildung 2). Da erscheint es logisch, dass diese Moleküle nicht nur die Cholesterin-, sondern auch die Coenzym-Q10-Synthese hemmen. Die meisten bisherigen Studien, in denen die Interaktionen zwischen Coenzym Q1ound der Einnahme von Statinen untersucht wurden, bestätigen dies.

In einer prospektiven Studie wurde beobachtet, dass sich bei den mit Simvastatin behandelten Patienten mit Hypercholesterinämie das LDL-Cholesterin, das Gesamtcholesterin und der Plasmaspiegel von Coenzym Q10 deutlich reduzierten (Coenzym Q10 von 1.08 auf $0.80 \mathrm{mg} / \mathrm{dl}$ ) [7]. WATTS et al [8] beschrieben ähnliche Resultate, wie auch RUNDEK et al. mit Atorvastatin [8a]. Auch unter der Behandlung mit Pravastatin und Lovastatin wurde eine ausgeprägte Reduktion der Plasmaspiegel von Coenzym Q10 festgestellt [9]. In einer weiteren Studie mit 12 Freiwilligen erhielten die Probanden während vier Wochen 20 mg Pravastatin; es konnten keine Unterschiede bei den Coenzym-Q10-Spiegeln beobachtet werden [10]. Bei Patienten mit Diabetes mellitus (nicht insulinabhängig), mit oder ohne Simvastatin-The-

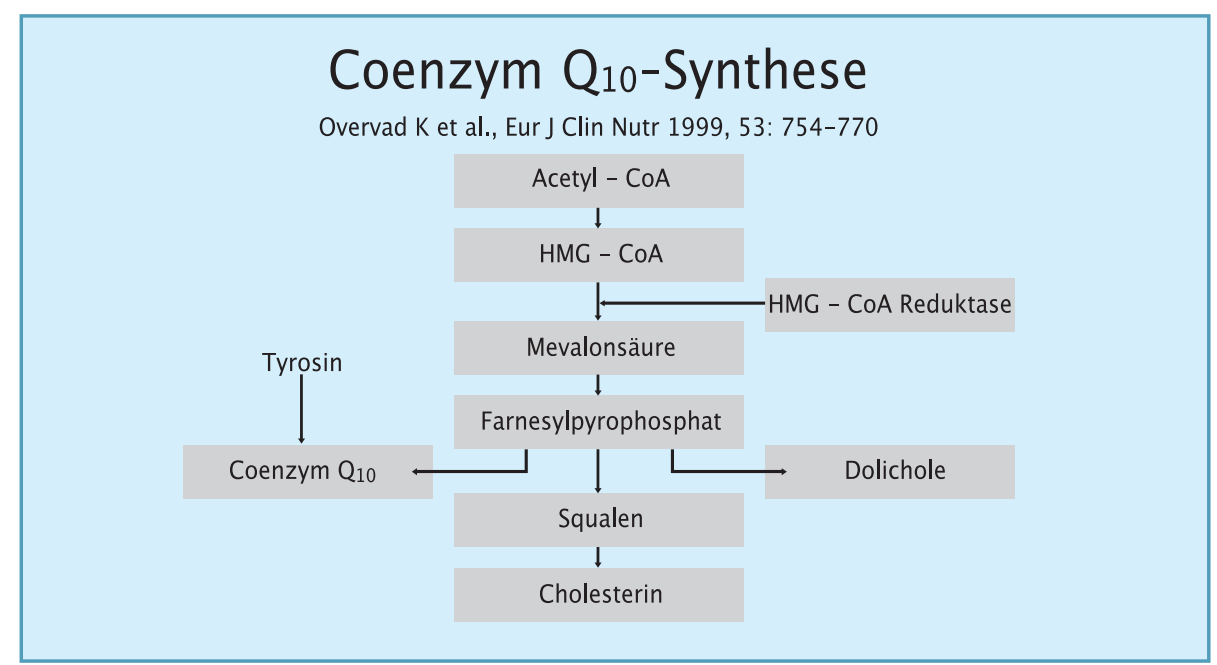

Abb. 2. Synthese von Coenzym $\mathrm{Q}_{10}$.

Tab. 1. Konzentration von Coenzym $\mathrm{Q}_{10}$ in den Zellmembranen und im Verhältnis zu alpha-Tocopherol [CRANE et al., op.cit.]

\begin{tabular}{lll} 
Membranen in der Rattenleber & $\mathbf{Q}_{10} /$ Protein $\boldsymbol{\mu g} / \mathbf{~ m g}$ & $\mathbf{Q}_{10} /$ Tocopherol mol / mol \\
\hline Mitochondrien (innere Membran) & 1.9 & 35 \\
\hline Zellmembranen & 0.7 & 21 \\
\hline Peroxisomen & 0.3 & 3 \\
\hline Lysosomen & 1.9 & 3 \\
\hline Golgi-Membran & 2.6 & 1 \\
\hline Endoplasmatisches Retikulum & 0.2 & 1 \\
\hline
\end{tabular}

Tab. 2. Coenzym $\mathrm{Q}_{10}$ in Nahrungsmitteln

\begin{tabular}{|lllll|}
\hline Nahrung & $\begin{array}{l}\text { Gehalt an } \\
\text { Coenzym } \\
\mathbf{Q}_{10}(\boldsymbol{\mu g} / \mathbf{g})\end{array}$ & $\begin{array}{l}\text { Durchschnittliche } \\
\text { Portion }(\mathbf{g} / \text { Tag) }\end{array}$ & $\begin{array}{l}\text { Menge an ein- } \\
\text { genommenem } \\
\text { Coenzym } \mathbf{Q}_{10} \text { (mg/Tag) }\end{array}$ \\
\hline Fleisch & Pouletschenkel & 17 & 120 & 2 \\
\hline & Rindsleber & 19 & 120 & 2.3 \\
\hline Fisch & Lering & 2.9 & 120 & 3.5 \\
\hline & Forelle & 11 & 26 & 0.7 \\
\hline
\end{tabular}

rapie, sind die Coenzym-Q10-Spiegel reduziert [11] genauso wie bei Patienten mit koronarer Herzkrankheit, die mit Pravastatin behandelt werden [12].

Coenzym Q10 kann auch mit anderen Substanzen interagieren [13]. In wenigen Einzelfällen wurde berichtet, dass es nach Gabe von Coenzym Q10 zu einer verstärkten Wirkung von Coumarinderivaten gekommen ist. Coenzym Q10 kann zudem die potenzielle Toxizität von Adriamycin und Doxorubicin verstärken, indem es den Abbau dieser
Substanzen hemmt. Möglicherweise wird die Wirksamkeit einer Strahlentherapie durch Coenzym Q10 herabgesetzt.

\section{Coenzym $\mathbf{Q}_{10}$ bei Herzerkrankungen}

Das Herz hat einen kontinuierlich hohen Energiebedarf und ist ständig oxidativ belastet. Aus diesem Grund wird Coenzym $\mathrm{Q}_{10}$ in der Therapie 
Tab. 3. Faktoren, welche die Coenzym $\mathrm{Q}_{10}$-Spiegel im Blut beeinflussen [35] (Probanden: $n=518,40-75$ Jahre, Männer und Frauen)

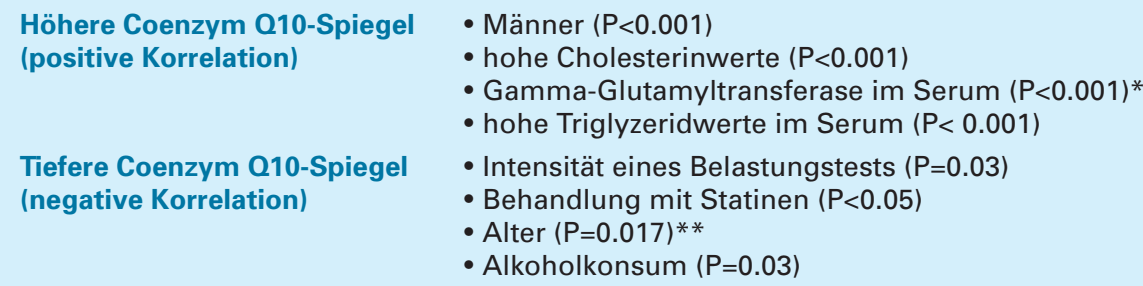

(1)

- Männer $(\mathrm{P}<0.001)$

- hohe Cholesterinwerte $(P<0.001)$

- Gamma-Glutamyltransferase im Serum $(P<0.001)^{*}$

- hohe Triglyzeridwerte im Serum $(P<0.001)$

- Intensität eines Belastungstests ( $P=0.03)$

- Behandlung mit Statinen $(P<0.05)$

- Alter $(P=0.017)^{* *}$

- Alkoholkonsum (P=0.03)

*) Weist auf Leberschaden hin, bei dem auch mitochondriale Bestandteile freigesetzt werden.

**) Man beachte auch die geringere Muskelmasse im Alter. Von anderen Autoren wurde dieser Faktor nicht bestätigt.

bzw. Prävention von Herzerkrankungen verwendet. Gemäss verschiedener Autoren führt die Gabe von Coenzym Q10 zu einer signifikanten und klinisch relevanten Verbesserung von Herzfunktionen, zum Beispiel des Herz-Zeitvolumens, der Ejektionsfraktion, des Herzindex und des enddiastolischen Volumenindex.

LIN et al. zeigten in einer Placebokontrollierten Studie mit 22 Patienten, dass die Gabe von 200 mg Coenzym Q10 täglich während fünf bis sieben Tagen die ventrikulären Funktionen nach einer kardioplegischen Hypothermie signifikant verbesserte [14]. Die Coenzym Q10-Therapie hatte jedoch keinen Einfluss auf die Vorhoffunktionen.

Overvad et al. publizierten eine Zusammenfassung der relevanten Studien, die in den Jahren 1985 bis 1995 zum Thema Herzinsuffizienz erschienen waren (Tabelle 4) [15]. In diesen Studien schwankten die Tagesdosen zwischen 60 und $200 \mathrm{mg}$ Coenzym Q10, die Behandlungsdauer lag zwischen einem und zwölf Monaten. SoJA und MorTENSEN machten eine Metaanalyse von verschiedenen Studien, die im selben Zeitraum erschienen und mit Herzinsuffizienz-Patienten durchgeführt worden waren [16]. Der Einfluss auf verschiedene kardiologische Variablen bei diesen Patienten ist in Tabelle 5 zusammengefasst.

OvERVAD et al. publizierten auch eine Zusammenfassung der 1985 bis 1995 erschienenen relevanten, meist kleinen Studien zum Thema Angina pectoris (Tabelle 6). Die Tagesdosen schwankten in diesen Untersuchungen zwischen 30 und 1'500 mg Coenzym Q10, die Behandlungsdauer zwischen einer und vier Wochen.

Neuere Arbeiten bestätigten nur in einer von drei Studien die Wirksamkeit von Coenzym $Q_{10}$ bei Herzinsuffizienz. $\mathrm{Zu}$ beachten ist, dass in den negativen Studien von WATSON et al. und von KнаттA et al. die Patienten mit ACE-Hemmern und je nach Bedarf und Verträglichkeit auch mit BetaBlockern therapiert wurden [17]. Eine allfällige Wirkung von Coenzym Q10 wurde möglicherweise durch die Wirkung der modernen Basisbehandlung überlagert. Eine weitere Studie zeigt die Wirksamkeit von Coenzym Q10 bei isolierter systolischer Hypertonie. In einer Untersuchung aus Indien zeigt sich eine bedeutende Schutzwirkung von Coenzym Q10 nach akutem Herzinfarkt (Tabelle 7).

\section{Coenzym $\mathbf{Q}_{10}$ bei Neuro-Myopathien}

Coenzym Q10 ist für eine normale Funktion der Muskeln sehr wichtig. Eine Coenzym-Q10-Unterversorgung kann die Muskelfunktion beeinträchtigen, da den Muskeln zu wenig Energie zur Verfügung steht. Es gibt eine Reihe von seltenen, angeborenen Defekten in den Mitochondrien, die dazu führen, dass der Muskulatur nicht genügend Energie bereitgestellt wird. Bei derartigen Muskeldystrophien konnte in verschiedenen Studien der positive Effekt einer Coenzym-Q10-Supplementierung nachgewiesen werden. Die bisherigen Untersuchungen zeigen, dass bei Patienten mit Mitochondriendefekten auf je- den Fall ein Therapieversuch mit Coenzym Q10 unternommen werden sollte. $\mathrm{Zu}$ diesen Erkrankungen gehören:

- MELAS-Syndrom [18]: Patienten mit mitochondrialer Myopathie, Enzephalopathie, Laktatazidose und Strokeähnlichen Episoden

- myotonische Dystrophie (Curschmann-Steinert-Batten-Syndrom) [19]: Autosomal-dominant erbliche Krankheit der Muskulatur (Myopathie). Störung des Muskeltonus, vorwiegend der Fingerbeuger, der kleinen Handmuskeln (abgeschwächter Händedruck), der Kau- und Zungenmuskulatur (verwaschene Sprache), fortschreitende Dystrophie der Augen- und Lidmuskulatur (hängendes Lid) sowie häufig auch der mimischen und Nackenmuskulatur, Parästhesien, Haarausfall, Cataracta myotonica, Schilddrüsenunterfunktion etc.

- Lewy-Körperchen-Krankheit: degenerative Demenz mit Merkmalen der Parkinson-Krankheit [20]

- Diabetes mellitus mit Taubheit (Mutation des mitochondrialen t-RNS) [21] - familiäre zerebellare Ataxie [22].

Auch bei Patienten mit Chorea Huntington wurden tiefe Coenzym-Q10Plasmaspiegel festgestellt [23]. Chorea Huntington ist eine autosomal-dominant erbliche, hirnatrophische Form der Chorea mit chronisch fortschreitendem Verlauf. Neben Athetose oder Torsionsdystonie, Parästhesien und schwerwiegenden psychischen Veränderungen tritt eine hochgradige Demenz auf; die Therapie ist symptomatisch. Es besteht Grund zur Hoffnung, dass eine Behandlung mit Coenzym Q10 bei Chorea Huntington eine Wirkung hat. In einer Studie aus dem Jahr 2001 erhielten 347 Chorea Huntington-Patienten während 30 Monaten täglich 600 mg Coenzym Q10; dabei wurde eine leider nicht signifikante Verlangsamung der Progredienz der Erkrankung um 13 Prozent festgestellt [24].

Bei Patienten mit Parkinson-Syndrom oder vaskulärer Demenz hat man bisher keine Verminderung der Coenzym Q10 -Werte entdeckt [25]. In einer Pilotstudie mit 15 Parkinson-Patienten zeigte Coenzym $\mathrm{Q}_{10}$ in einer Dosierung von $200 \mathrm{mg} / \mathrm{d}$ denn auch keine Wir- 
Tab. 4. Doppelblinde, Placebo-kontrollierte Studien mit Coenzym $\mathrm{Q}_{10}$-Supplementen bei chronischer Herzinsuffizienz, 1985-1995

\begin{tabular}{|c|c|c|c|c|c|c|c|}
\hline Referenzen & Diagnosen & $\mathbf{n}$ & Design & $\begin{array}{l}\text { NYHA (bei } \\
\text { Studienbeginn) }\end{array}$ & $\begin{array}{l}\text { Coenzym } \mathbf{Q}_{10-} \\
\text { Dosis (mg/d) }\end{array}$ & $\begin{array}{l}\text { Behandlung } \\
\text { (Monate) }\end{array}$ & Ergebnisse \\
\hline $\begin{array}{l}\text { Langsjoen } \\
\text { et al. (1985) }\end{array}$ & DCM & 19 & Cross-over & III-IV & 100 & $2 \times 3$ & $\begin{array}{l}\text { EF +, verbesserte } \\
\text { körperliche } \\
\text { Leistung }\end{array}$ \\
\hline $\begin{array}{l}\text { Judy } \\
\text { et al. (1986) }\end{array}$ & DCM & 14 & Cross-over & IV & 100 & $2 \times 3$ & $\begin{array}{l}\mathrm{EF}+, \mathrm{SV}+, \mathrm{CO}+ \\
\mathrm{EDVI}+\end{array}$ \\
\hline $\begin{array}{l}\text { Permanetter } \\
\text { et al. (1989) }\end{array}$ & DCM & 25 & Cross-over & I-III & 100 & $2 \times 4$ & Keine Wirkung \\
\hline $\begin{array}{l}\text { Rossi } \\
\text { et al. (1991) }\end{array}$ & IHD & 20 & $\begin{array}{l}\text { Parallele } \\
\text { Gruppen }\end{array}$ & Unbekannt & 200 & 3 & $\begin{array}{l}\text { Arbeitsleistung +, } \\
\text { EF und CO unverän- } \\
\text { dert, Puls und Blut- } \\
\text { druck während Be- } \\
\text { lastung unverändert }\end{array}$ \\
\hline $\begin{array}{l}\text { Serra } \\
\text { et al. (1991) }\end{array}$ & $\begin{array}{l}\text { Verschiedene } \\
\text { Ursachen }\end{array}$ & 20 & Cross-over & II-III & 60 & $2 \times 1$ & $\begin{array}{l}\mathrm{SV}+, \mathrm{CO}+, \\
\text { Leistung + }\end{array}$ \\
\hline $\begin{array}{l}\text { Pogessi } \\
\text { et al. (1991) }\end{array}$ & $\begin{array}{l}\text { Verschiedene } \\
\text { Ursachen }\end{array}$ & 20 & Cross-over & II-III & 100 & $2 \times 2$ & $\begin{array}{l}\mathrm{EF}+\text {, FS +, } \\
\mathrm{PET} / \mathrm{LVET}+\text {, Puls } \\
\text { und Blutdruck un- } \\
\text { verändert, NYHA- } \\
\text { Klassen unverändert }\end{array}$ \\
\hline $\begin{array}{l}\text { Morisco } \\
\text { et al. (1993) }\end{array}$ & $\begin{array}{l}\text { Verschiedene } \\
\text { Ursachen }\end{array}$ & 641 & $\begin{array}{l}\text { Parallele } \\
\text { Gruppen }\end{array}$ & III-IV & 100 & 12 & $\begin{array}{l}\text { NYHA-Klasse }+ \\
\text { weniger Hospitali- } \\
\text { sationen, weniger } \\
\text { Linksherzinsuffizienz (?) }\end{array}$ \\
\hline $\begin{array}{l}\text { Morisco } \\
\text { et al. (1994) }\end{array}$ & $\begin{array}{l}\text { Verschiedene } \\
\text { Ursachen }\end{array}$ & 6 & Cross-over & II-IV & 150 & $2 \times 1$ & $\begin{array}{l}\text { EF während Belas- } \\
\text { tung }+, \mathrm{CO} \text { während } \\
\text { Belastung }+\end{array}$ \\
\hline $\begin{array}{l}\text { Hofman-Bang } \\
\text { et al. (1995) }\end{array}$ & $\begin{array}{l}\text { Verschiedene } \\
\text { Ursachen }\end{array}$ & 79 & Cross-over & II-IV & 100 & $2 \times 3$ & $\begin{array}{l}\text { Arbeitsleistung +, } \\
\text { EF unverändert, } \\
\text { Lebensqualität + }\end{array}$ \\
\hline
\end{tabular}

Tab. 5. Metaanalyse bezüglich Coenzym $\mathrm{Q}_{10}$ bei Herzinsuffizienz: berechnete Effektgrösse mit den entsprechenden prozentualen Differenzen sowie den Ergebnissen der statistischen Signifikanz und Homogenitätstest zwischen den Studien

\begin{tabular}{|c|c|c|c|c|}
\hline Herzparameter & Effektgrösse* & Prozente & $\begin{array}{l}\text { Statistische } \\
\text { Signifikanz }\end{array}$ & $\begin{array}{c}\text { Homogenität (Übereinstimmung } \\
\text { zwischen den Studien) }\end{array}$ \\
\hline Ejektionsfraktion & 1.37 & $+92 \%$ & $P<0.0001$ & - \\
\hline Schlagvolumen & 0.71 & $+76 \%$ & $P<0.005$ & + \\
\hline Herzminutenvolumen & 0.61 & $+73 \%$ & $\mathrm{P}<0.05$ & + \\
\hline Kardialer Index & 1.15 & $+87 \%$ & $P<0.0001$ & - \\
\hline Enddiastolisches Volumen (Index) & 1.23 & $-88 \%$ & $P<0.0001$ & - \\
\hline Systolische Zeitintervalle & 0.13 & $+55 \%$ & - & + \\
\hline Maximale Arbeitsleistung & 0.13 & $+55 \%$ & - & + \\
\hline
\end{tabular}

kung [26]. In einer Nachfolgestudie aus dem Jahr 2002 erhielten 80 Patienten im Initialstadium der Parkinson-Krankheit dagegen hoch dosiertes Coenzym Q10 (bis zu 1'200 mg täglich), dabei konnte eine dosisabhängige Verlangsamung der Progression der Krankheit festgestellt werden [27]. Ein Plateau der Coenzym Q10-Spiegel wird allerdings erst bei 2’400 mg täglich erreicht [27a].
In einer offenen Studie war Coenzym Q10 angeblich auch wirksam als Intervalltherapie bei Migräne [28] sowie in Kombination mit Ginkgo biloba bei Fibromyalgie [29]. 
Tab. 6. Doppelblinde, Placebo-kontrollierte Studien mit Coenzym Q ${ }_{10}$-Supplementen bei Angina pectoris, 1985-1995

\begin{tabular}{|c|c|c|c|c|c|c|}
\hline Referenzen & Diagnosen & Demographie & Design & $\begin{array}{l}\text { Coenzym } \mathbf{Q}_{10-} \\
\text { Dosis }\end{array}$ & Dauer & Ergebnisse \\
\hline $\begin{array}{l}\text { Hiasa } \\
\text { et al. (1984) }\end{array}$ & $\begin{array}{l}\text { Stabile Angina } \\
\text { pectoris, } \\
\text { NYHA II }\end{array}$ & $\begin{array}{l}\mathrm{n}=18 \text { (13 Männer, } \\
5 \text { Frauen) } \\
\text { Alter: } 58 \pm 9 \text { Jahre }\end{array}$ & $\begin{array}{l}\text { Zwei Gruppen } \\
\text { parallel }\end{array}$ & $\begin{array}{l}1.5 \mathrm{mg} / \mathrm{kg} / \mathrm{d} \text {, } \\
\text { intravenös }\end{array}$ & 7 Tage & $\begin{array}{l}\text { Verbesserte Arbeitstole- } \\
\text { ranz, Tendenz zu weniger } \\
\text { ST-Depressionen, } \\
\text { Puls und Blutdruck } \\
\text { unverändert }\end{array}$ \\
\hline $\begin{array}{l}\text { Kamikawa } \\
\text { et al. (1985) }\end{array}$ & $\begin{array}{l}\text { Stabile } \\
\text { Angina pectoris }\end{array}$ & $\begin{array}{l}\mathrm{n}=12(10 \text { Männer, } \\
2 \text { Frauen) } \\
\text { Alter: } 50 \pm 6 \text { Jahre }\end{array}$ & Cross-over & $\begin{array}{l}150 \text { mg/d, } \\
\text { Kapseln }\end{array}$ & 4 Wochen & $\begin{array}{l}\text { Verbesserte Arbeitstole- } \\
\text { ranz, Tendenz zu weniger } \\
\text { ST-Depressionen, Puls } \\
\text { und Blutdruck unverän- } \\
\text { dert, zu weniger Angina } \\
\text { pectoris-Anfällen und } \\
\text { Abnahme des Nitrogly- } \\
\text { cerinverbrauchs }\end{array}$ \\
\hline $\begin{array}{l}\text { Schardt } \\
\text { et al. (1986) }\end{array}$ & $\begin{array}{l}\text { Stabile } \\
\text { Angina pectoris }\end{array}$ & $\begin{array}{l}\mathrm{n}=15 \text { (3 Männer, } \\
12 \text { Frauen) } \\
\text { Alter: } 50-60 \text { Jahre }\end{array}$ & Cross-over & $\begin{array}{l}600 \mathrm{mg} / \mathrm{d} \text {, } \\
\text { Kapseln }\end{array}$ & 4 Tage & $\begin{array}{l}\text { Weniger ST-Depressio- } \\
\text { nen, verminderter } \\
\text { systolischer Blutdruck, } \\
\text { Puls und diastolischer } \\
\text { Blutdruck unverändert }\end{array}$ \\
\hline $\begin{array}{l}\text { Mazzola } \\
\text { et al. (1991) }\end{array}$ & $\begin{array}{l}\text { Stabile Angina } \\
\text { pectoris und } \\
\text { leichte bis } \\
\text { mittelschwere } \\
\text { Herzinsuffizienz }\end{array}$ & $\begin{array}{l}\mathrm{n}=20 \text { (11 Männer, } \\
9 \text { Frauen) } \\
\text { Alter: } 59 \pm 7 \text { Jahre }\end{array}$ & Cross-over & $\begin{array}{l}60 \mathrm{mg} / \mathrm{d} \\
\text { Kapseln }\end{array}$ & 4 Wochen & $\begin{array}{l}\text { Verbesserte Arbeitstole- } \\
\text { ranz, weniger Angina } \\
\text { pectoris-Anfälle, vermin- } \\
\text { derter Nitroglycerinver- } \\
\text { brauch, Puls und Blut- } \\
\text { druck unverändert }\end{array}$ \\
\hline $\begin{array}{l}\text { Wilson } \\
\text { et al. (1991) }\end{array}$ & $\begin{array}{l}\text { Stabile } \\
\text { Angina pectoris }\end{array}$ & $\begin{array}{l}\mathrm{n}=58 \\
\text { (hauptsächlich } \\
\text { Männer) } \\
\text { Alter: durchschnittlich } \\
60 \text { Jahre }\end{array}$ & $\begin{array}{l}\text { Drei Gruppen } \\
\text { parallel }\end{array}$ & $\begin{array}{l}30 \mathrm{mg} / \mathrm{d} \text {, } \\
150 \mathrm{mg} / \mathrm{d} \\
\text { Kapseln }\end{array}$ & 4 Wochen & $\begin{array}{l}\text { Verbesserte Arbeitstole- } \\
\text { ranz (dosisunabhängig), } \\
\text { unveränderter Nitrogly- } \\
\text { cerinverbrauch, unverän- } \\
\text { derter Puls und Blutdruck }\end{array}$ \\
\hline
\end{tabular}

Tab. 7. Neuere doppelblinde, Placebo-kontrollierte Studien mit Coenzym $\mathrm{Q}_{10}$-Supplementen bei kardiovaskulären Krankheiten, $1998-2002$

\begin{tabular}{|c|c|c|c|c|c|c|}
\hline Autor & $\mathbf{n}$ & Design & $\begin{array}{l}\text { Coenzym } \mathrm{Q}_{10^{-}} \\
\text {Dosis (mg/Tag) }\end{array}$ & Dauer & $\begin{array}{l}\text { Indikation und Variablen } \\
\text { Akuter Myokardinfarkt: }\end{array}$ & Resultat \\
\hline $\begin{array}{l}\text { Singh RB, } \\
1998^{36}\end{array}$ & 143 & DBPG & 120 & 28 Tage & $\begin{array}{l}\text { 1. Angina pectoris } \\
\text { 2. Totale Arrhythmien } \\
\text { 3. Linksventrikuläre Insuffizienz } \\
\text { 4. Kardiale Ereignisse (inkl. Tod) }\end{array}$ & $\begin{array}{l}\text { 1. } 9.5 \text { vs. } 28.1 \% \\
\text { 2. } 9.5 \text { vs. } 25.3 \% \\
\text { 3. } 8.2 \text { vs. } 22.5 \% \\
\text { 4. } 15.0 \text { vs. } 30.9 \%\end{array}$ \\
\hline $\begin{array}{l}\text { Munkholm H, } \\
1999^{37}\end{array}$ & 22 & DBPG & 100 & 3 Monate & $\begin{array}{l}\text { Linksherzinsuffizienz } \\
\text { - Basal und nach Belastung: } \\
\text { Schlagindex } \\
\text { - Druck und Verschlussdruck } \\
\text { in der Arteria pulmonalis }\end{array}$ & $\begin{array}{l}\text { Besserung } \\
\text { der Variablen }\end{array}$ \\
\hline $\begin{array}{l}\text { Watson PS, } \\
199938\end{array}$ & 27 & DBCO & 100 & 3 Monate & $\begin{array}{l}\text { Herzinsuffizienz } \\
\text { - Linksventrikuläre } \\
\text { Ejektionsfraktion } \\
\text { - Herzvolumen } \\
\text { - Hämodynamische Indizes } \\
\text { - Gesundheitsbezogene } \\
\text { Lebensqualität }\end{array}$ & Kein Einfluss \\
\hline $\begin{array}{l}\text { Khatta M, } \\
1999^{39}\end{array}$ & 47 & DBPG & 200 & 6 Monate & $\begin{array}{l}\text { Herzinsuffizienz } \\
\text { NYHA III und IV } \\
\text { - Linksventrikuläre Ejektionsfraktion } \\
\text { - Maximaler Sauerstoffverbrauch } \\
\text { - Ergometriedauer }\end{array}$ & Kein Einfluss \\
\hline $\begin{array}{l}\text { Burke BE, } \\
2001^{40}\end{array}$ & 83 & DBPG & 120 & 3 Monate & $\begin{array}{l}\text { Isolierte systolische Hypertonie } \\
\text { Blutdruckreduktion }\end{array}$ & $\begin{array}{l}\text { Systolischer Druck um } \\
18 \mathrm{mmHg} \text { signifikant } \\
\text { gesenkt }\end{array}$ \\
\hline
\end{tabular}

Abkürzungen: DBPG = doppelblind, parallele Gruppen; DBCO = doppelblind, cross-over 
Tab. 8. Coenzym $\mathrm{Q}_{10}$ zur Diabetesbehandlung

\begin{tabular}{|c|c|c|c|c|c|c|}
\hline Autor & $\mathbf{n}$ & Design- & $\begin{array}{l}\text { Coenzym Q10- } \\
\text { Dosis (mg/Tag) }\end{array}$ & Dauer & Variablen & Resultat \\
\hline $\begin{array}{l}\text { Henriksen JE, } \\
1999\end{array}$ & 34 & DBPG & 100 & 3 Monate & $\begin{array}{l}\text { Diabetes Typ 1: Insulindosis, HbA1c, } \\
\text { Hypoglykämien, gesundheitsbezogene } \\
\text { Lebensqualität }\end{array}$ & Kein Einfluss \\
\hline $\begin{array}{l}\text { Watts GF, } \\
2002\end{array}$ & 40 & DBPG & 200 & 3 Monate & $\begin{array}{l}\text { Diabetes Typ } 2 \text { (NIDDM) und } \\
\text { Dyslipidämien: Dilatation der A. brachialis } \\
\text { (Endothel-abhängig und -unabhängig); } \\
\text { HbA1c, Glykämie }\end{array}$ & $\begin{array}{l}\text { Zunahme der } \\
\text { Dilatation; kein } \\
\text { Einfluss auf die } \\
\text { restlichen Variablen }\end{array}$ \\
\hline
\end{tabular}

Tab. 9. Coenzym $\mathrm{Q}_{10}$ in der Sportmedizin zur Leistungssteigerung

\begin{tabular}{|c|c|c|c|c|c|c|}
\hline Autor & $\mathbf{n}$ & Design & $\begin{array}{l}\text { Coenzym } \mathbf{Q}_{10-} \\
\text { Dosis }\end{array}$ & Dauer & Variablen & Resultat \\
\hline $\begin{array}{l}\text { Weston SB, } \\
1997\end{array}$ & 18 & DBPG & $1 \mathrm{mg} / \mathrm{kg} / \mathrm{Tag}$ & 28 Tage & $\begin{array}{l}\text { Sportler: Sauerstoffaufnahme, } \\
\text { Laktatspiegel, Glukose- und } \\
\text { Triglyzeridkinetik, Herzfrequenz } \\
\text { und Blutdruck während } \\
\text { Belastung bis Erschöpfung }\end{array}$ & Kein Einfluss \\
\hline $\begin{array}{l}\text { Kaikkonen J, } \\
1998\end{array}$ & 37 & DBPG & $\begin{array}{l}90 \mathrm{mg} / \mathrm{Tag} \\
\text { plus Vitamin E }\end{array}$ & 21 Tage & $\begin{array}{l}\text { Vorbehandlung von Marathonläufern: } \\
\text { Lipidperoxidation, CK-Spiegel, } \\
\text { Laktatspiegel }\end{array}$ & Kein Einfluss \\
\hline $\begin{array}{l}\text { Ylikoski T, } \\
1997\end{array}$ & 25 & $\mathrm{DBCO}$ & $90 \mathrm{mg} / \mathrm{Tag}$ & & $\begin{array}{l}\text { Langläufer (Cross-Country): } \\
\text { Leistungsvariablen und subjektive } \\
\text { Befindlichkeit }\end{array}$ & $\begin{array}{l}\text { Subjektive Befind- } \\
\text { lichkeit: } \\
\text { bei Coenzym } \mathrm{Q}_{10} \\
\text { um } 94 \% \text { besser } \\
\text { vs. } 33 \% \text { bei Placebo }\end{array}$ \\
\hline
\end{tabular}

\section{Coenzym $\mathbf{Q}_{10}$ bei anderen Indikationen}

Coenzym $Q_{10}$ wurde bei Patienten mit Diabetes mellitus Typ 1 oder Typ 2 weitgehend ergebnislos untersucht (Tabelle 8) [30, 31].

In zwei von drei Studien, in denen die Wirkung von Coenzym $\mathrm{Q}_{10}$ bei Sportlern untersucht wurde, konnte durch die Verabreichung von Coenzym Q10 keine Steigerung der Leistung erzielt werden [32,33]. In der dritten Studie zeigte sich eine subjektive Besserung (Tabelle 9) [34].

\section{Schlussfolgerungen}

\section{Allgemeines}

Bei verschiedenen Erkrankungen und im Alter werden tiefe Coenzym-Q10Blutspiegel gemessen. Daraus lässt sich aber kein physiologisch bedeutsamer Mangel bzw. die Notwendigkeit einer Supplementation direkt ableiten.

Statine senken (wahrscheinlich) die Coenzym-Q10-Spiegel. Dies kann mit Coenzym-Q10-Supplementen korrigiert werden; der klinische Nutzen ist allerdings nicht erwiesen. Die Behandlung mit Squalestatin erhöht hingegen die Coenzym-Q10-Spiegel um das Drei- bis Vierfache.

\section{Supplementation}

Bei einer Coenzym-Q10-Supplementation beträgt die Tagesdosis bis $30 \mathrm{mg}$. Die Tagesdosis im therapeutischen Einsatz beträgt zwischen 60 und 1'200 mg und ist anhand der publizierten Studien empirisch nach Indikation festzulegen. Dosisfindungsstudien liegen nicht vor, ausser bei der Parkinson-Krankheit.

Die galenische Form bzw. die Zubereitung eines Coenzym-Q10-Supple- ments kann die Bioverfügbarkeit sehr stark beeinflussen.

Da Coenzym Q10 Teil der Atmungskette ist, scheint die Kombination von Coenzym Q10 mit anderen Radikalfängern wie Vitamin C, Vitamin E, Selen etc. sinnvoll.

\section{Indikationen}

Coenzym-Q10-Supplemente sind bei mitochondrialen enzephalomyopathischen Leiden (z.B. MELAS) und bei hereditärem Diabetes mellitus mit Taubheit indiziert.

Die Therapie mit Coenzym Q10 bei Herzinsuffizienz, Angina pectoris und Kardiomyopathien kann in Kombination mit der Standardtherapie sinnvoll sein.

Es scheint wahrscheinlich, dass die Gabe von hoch dosiertem Coenzym Q 10 (bis zu 1'200 mg täglich) die Progression der Parkinson-Krankheit im Anfangsstadium verlangsamen kann.

Die Wirkung einer Coenzym-Q10- 
Supplementation bei anderen Indikationen, zum Beispiel zur Behandlung von Diabetikern (Typ 1 oder 2), zur Leistungssteigerung in der Sportmedizin oder zur rascheren Wundheilung bei geschwächten Patienten, ist noch ungenügend belegt.

This paper is an updated summary of an expert-workshop sponsored by BIOMED AG, $\mathrm{CH}$-Dübendorf.

\section{Literatur}

1) Shults $C W$, Haas RH, Passov D, Beal MF: Coenzyme Q10 levels correlate with the activities of complexes | and |I/III in mitochondria from parkinsonian and nonparkinsonian subjects. Ann Neurol (1997 Aug) 42(2): 261-4

2) Crane FL: Biochemical functions of coenzyme Q10. J Am Coll Nutr (2001 Dec) 20(6): 591-8

3) van de Vijver LP, Weber C, Kardinaal AF, Grobbee DE, Princen HM, van Poppel G: Plasma coenzyme Q10 concentrations are not decreased in male patients with coronary atherosclerosis. Free Radic Res (1999 Mar) 30(3): 165-72

4) Wolters $M$, Hahn A: Plasma ubiquinone status and response to six-month supplementation combined with multivitamins in healthy elderly women - results of a randomized, double-blind, placebo-controlled study. Int J Vitam Nutr Res 2003 May 73(3): 207-14

5) Kaikkonen J, Tuomainen TP, Nyyssonen K, Salonen JT: Coenzyme Q10: absorption, antioxidative properties, determinants, and plasma levels. Free Radic Res (2002 Apr) 36(4): 389- 97

6) Chopra RK, Goldman R, Sinatra ST, Bhagavan $\mathrm{HN}$ : Relative bioavailability of coenzyme Q10 formulations in human subjects. Int $\mathrm{J}$ Vitam Nutr Res (1998) 68(2): 109-113

7) Bargossi $A M$, Battino $M$, Gaddi $A$, Fiorella $P L$, Grossi G, Barozzi G, Di Giulio R, Descovich G, Sassi S, Genova ML et al. Exogenous Coenzym Q10 preserves plasma ubiquinone levels in patients treated with 3-hydroxy-3-methylglutaryl coenzyme. A reductase inhibitors. Int J Clin Lab Res (1994) 24(3): 171-176

8) Watts GF, Castelluccio C, Rice-Evans C, Taub NA, Baum H, Quinn PJ: Plasma coenzyme Q (ubiquinone) concentrations in patients treated with simvastatin. J Clin Pathol (1993 Nov)

8a) Rundek T, Naini A, Sacco R, Coates K, DiMauro S: Atorvastatin decreases the coenzyme Q10 level in the blood of patients at risk for cardiovascular disease and stroke. Arch Neurol (2004 Jun) 61(6):889-92

9) Mortensen SA, Leth A, Agner E, Rohde M: Dose-related decrease of serum coenzyme Q10 during treatment with HMG-COA reductase inhibitors. Mol Aspects Med (1997) 18 Suppl: S137-44

10) Bleske BE, Willis RA, Anthony M, Casselberry $N$, Datwani M, Uhley VE, Secontine SG, Shea MJ: The effect of pravastatin and atorvastatin on coenzyme Q10. Am Heart J (2001 Aug) 142(2): E2

11) Miyake $Y$, Shouzu $A$, Nishikawa $M$, Yonemoto $T$, Shimizu H, Omoto S, Hayakawa T, Inada M: Effect of treatment with 3-hydroxy-3-methylglutaryl coenzyme. A reductase inhibitors on serum coenzyme $\mathrm{Q} 10$ in diabetic patients. Arzneimittelforschung (1999 Apr) 49(4): 324-9

12) Hanaki $Y$, Sugiyama $S$, Ozawa $T$, Ohno $M$ : Coenzyme Q10 and coronary artery disease. Clin Investig (1993) 71(8 Suppl):S112-5

13) Jones K, Hughes K, Mischley L, McKenna DJ: Coenzyme Q-10: efficacy, safety, and use. In: Altern Ther Health Med (2002 May-Jun) 8(3): 42-55; quiz 56, 138
14) Chen YF Lin YT, Wu SC: Effectiveness of coenzyme Q10 on myocardial preservation during hypothermic cardioplegic arrest. J Thorac Cardiovasc Surg (1994 Jan) 107(1): 242-7

15) Overvad K, Diamant B, Holm L, Holmer G, Mortensen SA, Stender S: Coenzyme Q10 in health and disease. Eur J Clin Nutr (1999 Oct) 53(10): 764-70

16) Soja AM, Mortensen SA: Treatment of congestive heart failure with coenzyme Q10 illuminated by meta-analyses of clinical trials. Mol Aspects Med (1997) 18 Suppl: S159-68

17) Raj $S R$, Weisel RD, Verma S: Coenzyme $Q(10)$ and congestive heart failure: what is the verdict? Can J Cardiol (2002 Oct) 18(10): 1054-8

18) Abe K, Matsuo $Y$, Kadekawa J, Inoue S, Yanagihara T: Effect of coenzyme Q10 in patients with mitochondrial myopathy, encephalopathy, lactic acidosis, and stroke-like episodes (MELAS): evaluation by noninvasive tissue oximetry. J Neuro Sci (1999 Jan 1) 162(1): 65-8

19) Siciliano G, Mancuso M, Tedeschi $D$, Manca ML, Renna MR, Lombardi V, Rocchi A, Martelli F, Murri L: Coenzyme Q10, exercise lactate and CTG trinucleotide expansion in myotonic dystrophy. Brain Res Bull (2001 Oct-Nov 1) 56(3-4): 405-410

20) Molina JA, de Bustos F, Ortiz $S$, Del Ser T, Seijo M, Benito-Leon J, Oliva JM, Perez S, Manzanares J: Serum levels of coenzyme $\mathrm{Q}$ in patients with Lewy body disease. J Neural Transm (2002 Sep) 109(9): 1195-201

21) Suzuki $Y$, Taniyama $M$, Muramatsu $T$, Atsumi $Y$, Hosokawa K, Asahina T, Shimada A, Murata C, Matsuoka K: Diabetes mellitus associated with 3243 mitochondrial tRNA(Leu(UUR))mutation: clinical features and coenzyme Q10 treatment. Mol Aspects Med (1997) 18 Suppl: S181-8

22) Musumeci $O$, Naini $A$, Slonim $A E$, Skavin $N$, Hadjigeorgiou GL, Krawiecki N Weissman BM, Tsao CY, Mendell JR, Shanske S, De Vivo DC Hirano M, Di Mauro S: Familial cerebellar ataxia with M, Di Mauro S: Familial cerebellar ataxia with
muscle coenzyme Q10 deficiency. Neurology (2001 Apr 10) 56(7): 849-55

23) Koroshetz WJ, Jenkins BG, Rosen BR, Beal MF: Energy metabolism defects in Huntington's disease and effects of coenzyme Q10. Ann Neuro (1997 Feb) 41(2): 160-5

24) A randomized, placebo-controlled trial of coenzyme Q10 and remacemide in Huntington's disease. Neurology (2001 Aug 14) 57(3): 397-404

25) Jimenez FJ, Molina JA de Bustos F, GarciaRedondo A, Gomez-Escalonilla C Martinez-Salio A, Berbel A, Camacho A, Zurdo M, Barcenilla B, Enriquez de Salamanca R, Arenas J: Serum levels of coenzyme Q10 in patients with Parkinson's disease. J Neural Transm (2000) 107(2): $177-81$

26) Shults CW, Beal MF, Fontaine $D$, Nakano K, Haas $\mathrm{RH}$ : Absorption, tolerability, and effects on mitochondrial activity of oral coenzyme Q10 in parkinsonian patients. Neurology (1998 Mar) 50(3): 793-5

27) Shults CW, Oakes $D$, Kieburtz K, Beal MF, Haas R, Plumb S, Juncos JL, Nutt J, Shoulson I, Carter J, Kompoliti K, Perlmutter JS, Reich S Stern M, Watts RL, Kurlan R, Molho E, Harrison M, Lew $\mathrm{M}$ : Effects of coenzyme Q10 in early Parkinson disease: evidence of slowing of the functional decline. Arch Neurol (2002 Oct) 59(10): 1541-50

27a) Shults CW, Flint Beal $M$, Song $D$, Fontaine $D$ : Pilot trial of high dosages of coenzyme Q10 in patients with Parkinson's disease. Exp Neurol (2004 Aug) 188(2):491-4

28) Rozen TD, Oshinsky ML, Gebeline CA, Bradley KC Young WB Shechter AL, Silberstein SD: Open label trial of coenzyme $\mathrm{Q} 10$ as a migraine Open label trial of coenzyme Q10 as a migraine
preventive. Cephalalgia (2002 Mar) 22(2): preventive.
$137-41$

29) Lister RE: An open, pilot study to evaluate the potential benefits of coenzyme Q10 combined with Ginkgo biloba extract in fibromyalgia synwith Ginkgo biloba extract in fibromyalgia syn-
drome. J Int Med Res (2002 Mar-Apr) 30(2): 195-9

30) Henriksen JE, Andersen CB, Hother-Nielsen $O$ Vaag A, Mortensen SA, Beck-Nielsen H: Impact of ubiquinone (coenzyme Q10) treatment on glycaemic control, insulin requirement and wellbeing in patients with Type 1 diabetes mellitus. Diabet Med (1999 Apr) 16(4): 312-8

31) Watts GF Playford $D A$, Croft $K D$, Ward NC, Mori TA, Burke V: Coenzyme Q(10) improves endothelial dysfunction of the brachial artery in Type II diabetes mellitus. Diabetologia (2002 Mar) 45(3): 420-6

32) Weston SB, Zhou S, Weatherby RP, Robson SJ: Does exogenous coenzyme Q10 affect aerobic capacity in endurance athletes? Int J Sport Nutr (1997 Sep) 7(3): 197-206

33) Kaikkonen J, Kosonen L, Nyyssonen K, PorkkalaSarataho E, Salonen R, Korpela H, Salonen JT: Effect of combined coenzyme Q10 and d-alphatocopheryl acetate supplementation on exerciseinduced lipid peroxidation and muscular damage: a placebo-controlled double-blind study in marathon runners. Free Radic Res (1998 Jul) 29(1): thon runn

34) Ylikoski T, Piirainen J, Hanninen $O$, Penttinen J: The effect of coenzyme Q10 on the exercise performance of cross-country skiers. Mol Aspects Med (1997) 18 Suppl: S283-90

35) Kaikkonen J, Nyyssonen K, Tuomainen TP, Ristonmaa U, Salonen JT: Determinants of plasma coenzyme Q10 in humans. FEBS Lett (1999 Jan 25) $443(2): 163-6$

36) Singh $R B$, Wander GS, Rastogi $A$, Shukla $P K$ Mittal A, Sharma JP, Mehrotra SK, Kapoor R, Chopra RK: Randomized, double-blind placebocontrolled trial of coenzyme Q10 in patients with acute myocardial infarction. Cardiovasc Drugs Ther (1998 Sep) 12(4): 347-53

37) Munkholm $\mathrm{H}$, Hansen $\mathrm{HH}$, Rasmussen $\mathrm{K}$ : Coenzyme $\mathrm{Q} 10$ treatment in serious heart failure. Biofactors (1999) 9(2-4): 285-9

38) Watson PS, Scalia GM, Galbraith A, Burstow DJ, Bett N, Aroney CN: Lack of effect of coenzyme $\mathrm{Q}$ on left ventricular function in patients with congestive heart failure. J Am Coll Cardiol (1999 May) 33(6):1549-52

39) Khatta $M$, Alexander BS, Krichten $C M$, Fisher $\mathrm{ML}$, Freudenberger R, Robinson SW, Gottlieb SS: The effect of coenzyme $\mathrm{Q} 10$ in patients with SS: The effect of coenzyme 10 in patients with congestive heart failure.

40) Burke BE, Neuenschwander R, Olson RD: Randomized, double-blind, placebo-controlled trial of coenzyme Q10 in isolated systolic hypertension. South Med J (2001 Nov) 94(11): 1112-7

\section{Korrespondenzadresse:}

Prof. Dr. med. Reinhard Saller

UniversitätsSpital Zürich

Dep. für Innere Medizin

Institut für Naturheilkunde

Rämistrasse 100, CH-8091 Zürich

reinhard.saller@usz.ch 